\title{
New-Fangled Slowly-Absorbable versus Non-Absorbable Sutures for Abdominal Fascial Closure. Have the Goals towards an Advantageous Suture Been Met?
}

\author{
Georgios Anthimidis ${ }^{1}$, Marios Gregoriou ${ }^{1}$, Thomas Stavrakis ${ }^{1 *}$, Kalliopi Vasiliadou $^{1}$, Ioannis Lyras $^{2}$, \\ Kostas Ioannidis ${ }^{3}$, George Basdanis ${ }^{1}$ \\ ${ }^{1}$ Surgical Department, Interbalkan Medical Center of Thessaloniki, Thessaloniki, Greece \\ ${ }^{2}$ Clinic of Plastic Surgery of the Athens Medical Center, Athens, Greece \\ ${ }^{3}$ Surgical Clinic, General Hospital of Drama, Drama, Greece \\ Email: anthe_geo@mail.com,drgregoriou@gmail.com, "tstavrakis@gmail.com,lyras@lifting.gr, \\ arioann@yahoo.gr,gbasdanis@gmail.com
}

Received February 8, 2013; revised March 10, 2013; accepted March 18, 2013

Copyright (c) 2013 Georgios Anthimidis et al. This is an open access article distributed under the Creative Commons Attribution License, which permits unrestricted use, distribution, and reproduction in any medium, provided the original work is properly cited.

\begin{abstract}
Background-Aim: The technique of abdominal closure along with the material to be used is constantly evolving. The aim of the present study is to evaluate differences in midline laparotomy closure with a standard closure technique and new-fangled slow-absorbable versus non-absorbable sutures. Material and Methods: A prospective, comparative study of patients undergoing laparotomy closure with either STARDIOX (Polydioxanone) or STARLENE (Polypropylene) sutures during a 9 month period was performed. Patients were evaluated and compared in terms of surgical site infection, incisional hernia, burst abdomen, and suture sinus formation. Results: A total of 284 patients were included [141 in the STARDIOX (Polydioxanone) group and 143 in the STARLENE (Polypropylene) group]. Sinus formation was not noticed and no palpable knots were reported in both groups. Moreover burst abdomen was never encountered. Incisional hernia rates were similar for both suture materials: $n=6$ (4.3\%) for the STARDIOX (Polydioxanone) group and $\mathrm{n}=5$ (3.5\%) for the STARLENE (Polypropylene) group. There was not statistically significant relationship between the type of suture that was used and wound infection: $\mathrm{n}=5(3.5 \%)$ in the STARDIOX (Polydioxanone) group and $\mathrm{n}=6$ (4.2\%) in the STARLENE (Polypropylene) group. Complications did not occur in $96.1 \%$ of all patients. Conclusions: Our study suggests that there are no significant differences between these two new-fangled sutures. It seems that progress of suture materials has led to a step towards the goals of a beneficial suture and from then on complications of surgical wound closure should be merely a matter of operative technique.
\end{abstract}

Keywords: Abdominal Closure; Suture Material; Midline Laparotomy

\section{Introduction}

Complications of surgical wound closure significantly increase morbidity and mortality. Thus, the technique of abdominal closure with the material to be used remains a matter of discussion. Suture selection is dependent on the anatomic site, surgeon's preference, and the required suture characteristics.

For abdominal closure, significant benefit has been shown in using nonabsorbable or delayed absorbable suture.

Nonabsorbable suture is a superior choice because it retains its tensile strength; yet, it has been shown to have

"Corresponding author. an increase in reported incisional pain [1]. This has led many surgeons to opt for delayed-absorbable suture, which is able to retain its original tensile strength for some time and has been reported to have decreased suture pain $[2,3]$.

The aim of the present study is to evaluate differences in midline laparotomy closure with a standard closure technique and new-fangled slow-absorbable versus nonabsorbable sutures.

\section{Material and Methods}

A prospective, comparative study of patients undergoing laparotomy closure with either STARDIOX (Poly-dioxanone) or STARLENE (Polypropylene) sutures during a 
9 month period (1/1/2012-30/9/2012) was performed. All patients who underwent abdominal surgery for gastrointestinal disease through a midline incision were included. Exclusions criteria were patients who were being reoperated upon through the same incision and deaths unrelated to the wound. Patients were stratified according to age, sex, type of operation and degree of operative contamination and were randomly allocated to mass closure of the abdominal wall with continuous STARDIOX (Polydioxanone) or continuous STARLENE (Polypropylene) by the same surgeon. The skin was closed with clips; wound drains were not employed.

All patients received subcutaneous heparin; bowel preparation and antibiotic prophylaxis were given according to the surgeon's usual routine.

Postoperative follow-up was performed at 10 and 30 days (early), and 3 months (late); patients were evaluated and compared in terms of surgical site infection, incisional hernia, burst abdomen, and suture sinus formation. The patients were assessed by two "blinded" observers (surgeons).

All data were recorded using Statistical Package for the Social Sciences (SPSS) 16 for Windows for statistical analyses. As the main objective of the present study was to evaluate patients undergoing laparotomy closure with either STARDIOX (Polydioxanone) or STARLENE (Polypropylene) sutures, data were tabulated comparing between these two groups of patients in terms of surgical site infection, incisional hernia, burst abdomen, and suture sinus formation.

\section{Results}

A total of 284 patients were included [141 in the STARDIOX (Polydioxanone) group and 143 in the STARLENE (Polypropylene) group]. The comparability of the two groups is shown in Table 1.

The parameters were well matched. There was no statistically significant heterogeneity among the patient characteristics, wound classification, type of surgery and procedure.

The type, number and percentage of complications along with the statistical results are illustrated in Table 2 Complications did not occur in $96.1 \%$ of all patients.

Statistical comparisons were performed using the Chi-square test for independence for the categorical dependent variables. The assumptions that apply to the used techniques were not violated. $\mathrm{P}$ values below 0.05 were considered significant.

Sinus formation was not noticed and no palpable knots were reported in both groups. Moreover burst abdomen was never encountered. Complication rates were similar for both suture materials.

Particularly, there was no relationship between patients that suffered from incisional hernia and the type of
Table 1. Patient characteristics and type of surgery.

\begin{tabular}{ccc}
\hline & $\begin{array}{c}\text { STARDIOX } \\
\text { (Polydioxanone) } \\
\mathrm{n}=141\end{array}$ & $\begin{array}{c}\text { STARLENE } \\
\text { (Polypropylene) } \\
\mathrm{n}=143\end{array}$ \\
\hline PATIENTS & $60.5 \pm 15$ & $61.5 \pm 16$ \\
Age & 1.27 & 1.20 \\
Sex ration & & \\
FEMALE:MALE & 27 & 29 \\
SURGERY & 110 & 113 \\
Emergency & 12 & 10 \\
Clean & 19 & 20 \\
Clean/contaminated & & \\
Contaminated & 27 & 28 \\
PROCEDURE & 9 & 11 \\
Gastric & 105 & 104 \\
Small bowel & & \\
Colonic & &
\end{tabular}

suture that was used, since statistical comparison showed that the Pearson Chi-Square value (Yates' correction for continuity) was 0.001 , with an associated significance level of 0.981 .

Additionally, there was not statistically significant relationship between wound infection and type of suture that was used, as the Pearson Chi-Square value (Yates' correction for continuity) was 0.000 , with an associated significance level of 1.000 .

No differences were found in any of the follow-up assessments in any of the variables analyzed.

\section{Discussion}

The aims of wound closure include maintenance of tensile strength across the wound until tissue tensile strength is sufficient. Tissues should be held in proximity until adequate healing takes place to resist stress without mechanical support. Suture material is a foreign body implanted into human tissues; it educes a foreign body tissue reaction. Complications of wound healing, such as wound infection and incisional hernia, may be caused by patient factors (e.g., nutritional status), inappropriate suture choice, or a technique that causes extreme tension across the wound. Yet, no single material has provided all the characteristics of an ideal suture. In different conditions and with differentiation in tissue throughout the body, the requirements for satisfactory wound closure call for different suture characteristics.

As far as we could elicit from the literature, this clinical trial is the first to evaluate the effectiveness of STARDIOX (Polydioxanone, delayed-absorbable) versus STARLENE (Polypropylene, non-absorbable) for abdominal fascial closure in patients undergoing midline laparotomy incisions closed with a standard continuous 
Table 2. Type, number and percentage of complications.

\begin{tabular}{cccc}
\hline & STARDIOX (Polydioxanone) $\mathrm{n}=141$ & STARLENE (Polypropylene) $\mathrm{n}=143$ & $\mathrm{P}^{*}$ \\
\hline Incisional hernia & $\mathrm{n}=6(4.3 \%)$ & $\mathrm{n}=5(3.5 \%)$ & $0.981(>0.05)$ \\
Wound infection & $\mathrm{n}=5(3.5 \%)$ & $\mathrm{n}=6(4.2 \%)$ & $1.000(>0.05)$ \\
Sinus formation & $\mathrm{n}=0$ & $\mathrm{n}=0$ & \\
Burst abdomen & $\mathrm{n}=0$ & $\mathrm{n}=0$ & $1.000(>0.05)$ \\
Total & $\mathrm{n}=6(4.3 \%)$ & $\mathrm{n}=6(4.2 \%)$ & \\
\hline
\end{tabular}

$\mathrm{P}^{*}$ : significance level.

technique. The uniform effect of suture technique was reflected in the suture length to wound length ratio $(4: 1)$. The two groups were well matched for known risk factors. Patient assessment was "double-blind" as neither the examiner nor the patient knew which suture had been used.

Our results appear to be quite acceptable in accordance with the systematic review of Sajid et al. [4]. The fine results may be related to the physical characteristics and properties of the suture materials. Complication rates were similar for both groups. We had 11 incisional hernias in 284 laparotomies (3.9\%) even if they were not directly attributable to suture failure. Patients with incisional hernias were generally asymptomatic. The incidence of burst abdomen was zero.

Eight of these 11 incisional hernias followed colonic surgery (4 in each group). Seven of these 11 cases were emergent operations [4 with STARDIOX (Polydioxanone) and 3 with STARLENE (Polypropylene)]. Moreover 6 out of these 11 patients had a wound infection prior to the incisional hernia ( 3 in each group) and they had been classified as contaminated or clean/contaminated. Complications did not occur in clean wounds.

Although some incisional hernias do occur for up to 5 years postoperatively [5], it seems that most will be apparent at 3 months. However, the true incidence of incisional herniation will require longer follow-up.

Sinus formation did not develop in both groups, but it may do so in time.

We found that wounds closed with either STARDIOX (Polydioxanone) or STARLENE (Polypropylene) were generally comfortable; knots were not palpable and patients did not report wound pain.

From the results of our statistic, we can easily find that there are no differences between STARDIOX (Polydioxanone) and STARLENE (Polypropylene). However, there are some other differences between them. In particular, STARDIOX (Polydioxanone) is an absorbable suture composed of the polyester poly (p-dioxanone) with a reliable and predictable absorption. Our experience using STARDIOX (Polydioxanone) revealed superior pliability and smooth tissue passage. Yet, STAR LENE (Polypropylene) is a non-absorbable suture composed of a synthetic linear polyolefin, which elicits a minimal acute inflammatory reaction, followed by gradual encapsulation of the suture by fibrous tissue. Unlike absorbable sutures, STARLENE (Polypropylene) is not affected or weakened by tissue enzymes and conesquently is not subject to any degradation or loss of tensile strength. Moreover, STARLENE (Polypropylene) appears to be an extremely smooth textile, resulting in minimal tissue trauma and no tissue rupture, with outstanding elasticity, allowing for secured knot tying. It has been successfully implemented in contaminated and previously infected wounds as well.

As yet, infection remains the most important postoperative complication seeing as the incidence of incisional hernias may be decreased by the eradication of wound sepsis.

In conclusion, the selection of a specific suture material should be based on the patient, wound, tissue characteristics, and anatomic location. A surgeon's choice may not be based on scientific data, but rather, on the preferences that one has learned from mentors and/or during training. Comprehension of the individual characteristics of available suture materials is important to make an educated selection. Up to now, no one material is ideal and compromises have to be made. Our study suggests that STARDIOX (Polydioxanone) and STARLENE (Polypropylene) may be a step towards the goals of suture material and from then on complications of surgical wound closure could be merely a matter of operative technique. Since there are no significant differences between these two suture materials, additional studies may be conducted to estimate their cost-effectiveness and health-related quality of life.

\section{REFERENCES}

[1] J. Wissing, T. J. van Vroonhoven, M. E. Schattenkerk, et al., "Fascia Closure after Midline Laparotomy: Results of a Randomized Trial,” British Journal of Surgery, Vol. 74, No. 8, 1987, pp. 738-741. doi:10.1002/bjs.1800740831

[2] M. L. Corman, M. C. Veidenheimer and J. A. Coller, "Controlled Clinical Trial of Three Suture Materials for 
Abdominal Wall Closure after Bowl Operations," The American Journal of Surgery, Vol. 141, No. 4, 1981, pp. 510-513. doi:10.1016/0002-9610(81)90150-1

[3] B. M. Burt, A. Tavakkolizadeh and S. J. Ferzoco, "Incisions, Closures, and Management of the Abdominal Wound,” In: M. J. Zinner and S. W. Ashley, Eds., Maingot's Abdominal Operations, McGraw-Hill Companies, Inc., New York, 2007, p. 4.

[4] M. S. Sajid, U. Parampalli, M. K. Baig and M. R. McFall "A Systematic Review on the Effectiveness of Slowly-
Absorbable versus Non-Absorbable Sutures for Abdominal Fascial Closure Following Laparotomy,” International Journal of Surgery, Vol. 9, No. 8, 2011, pp. 615-625. doi:10.1016/j.ijsu.2011.09.006

[5] H. Ellis, H. Gajraj and C. D. George, "Incisional Hernias: When Do They Occur?” British Journal of Surgery, Vol. 70, No. 5, 1983, pp. 290-291. doi:10.1002/bjs. 1800700514 